Pure Appl. Chem., Vol. 84, No. 4, pp. 861-867, 2012.

http://dx.doi.org/10.1351/PAC-CON-12-01-10

(C) 2012 IUPAC, Publication date (Web): 11 March 2012

\title{
Putting aromatic compounds to work: Rational synthesis of organic 2D polymers*
}

\author{
A. Dieter Schlüter ${ }^{\ddagger}$ and Junji Sakamoto $\ddagger$ \\ Laboratory of Polymer Chemistry, Department of Materials, HCI J541, ETH Zurich, \\ Wolfgang-Pauli Strasse 10, $\mathrm{CH}-8093$ Zurich, Switzerland
}

\begin{abstract}
This contribution describes two different approaches aimed at the synthesis of monolayer molecular sheets with internal order, or, in other words, 2D polymers. First, an interfacial strategy is presented in which terpyridene-based hexafunctional monomers spread at the air/water interface are connected to one another with the help of metal salts. While this approach leads to micrometer-sized monolayer sheets that are mechanically stable enough to be spanned over several micrometer-sized holes, their internal structure could not yet be proven. The second approach rests upon solid-state photochemistry, and properly designed monomers are covalently connected with one another while being held in layered geometries owing to crystal packing. Exfoliation to single sheets can be achieved, and molecular structure is supported by a Raman spectroscopic analysis. We consider this the first case of a rational synthesis of $2 \mathrm{D}$ polymers and briefly touch on the impact this may have.
\end{abstract}

Keywords: aromatic compounds; molecular sheets; photochemistry; polymers; solid-state photochemistry.

\section{INTRODUCTION}

Monolayered covalent networks have always been a dream of synthetic chemists [1]. Already in 1935 the first synthesis was described in which a monolayer of compounds with polymerizable units was positioned at the air/water interface and cross-linked [2,3]. Many other examples followed, which all had in common that the internal structure of the network could not be controlled [1]. The recent discovery of graphene [4,5], which is a monolayered network with internal order, has further stimulated the interest in developing synthetic routes that would allow generating such networks with organic chemical means under ambient conditions and thus full structure control. According to a recent definition, such networks are referred to as 2D polymers [1]. They differ from the irregular networks reported so far by the presence of areal repeat units. In the 1920s, Staudinger coined the term "macromolecule" and described them as high-molar-mass chain compounds that consist of a linear array of (linear) repeat units [6]. 2D polymers with their areal repeat units can thus be considered an expansion of this fundamental concept. In this publication we describe two approaches toward solving this burning synthetic issue whereby one approach provides the first solution.

\footnotetext{
*Pure Appl. Chem. 84, 861-1112 (2012). A collection of invited papers based on presentations at the $14^{\text {th }}$ International Symposium on Novel Aromatic Compounds (ISNA-14), Eugene, OR, USA, 24-29 July 2011.

$\ddagger_{\text {Corresponding authors }}$
} 


\section{RESULTS AND DISCUSSION}

Strategies for how one could go about approaching 2D polymers were described in our 2009 review article [1]. They rest upon the two main factors, monomer design and pre-orientation of monomers into 2D prior to polymerization. For the many aspects that additionally need to be considered, the reader is referred to this review as well as a few other recent publications [7,8]. The 2009 review also contains the relevant literature in a rather comprehensive fashion that will therefore not be repeated here. For another brief review article and some more recent examples that, however, do not provide a full solution to the 2D polymer problem, see ref. [9] and refs. [10-17], respectively. Figure 1 shows the two main factors in a graphical representation. We propose to use shape-persistent, typically tri- or hexafunctional monomers which are either spread at the air/water interface or crystallized into laminar single crystals. Polymerization is achieved either by adding connector units from the subphase or by photochemical treatment, respectively.

Directed functionalities:

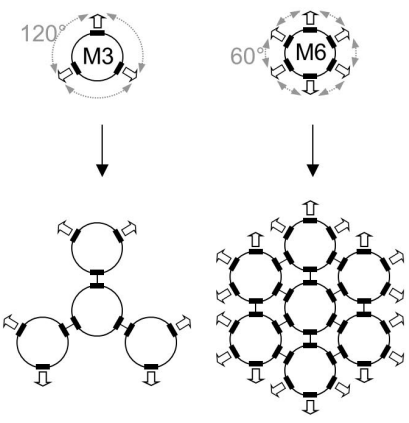

Confining into 2D:

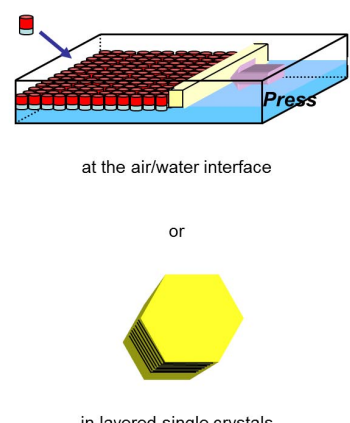

in layered single crystals

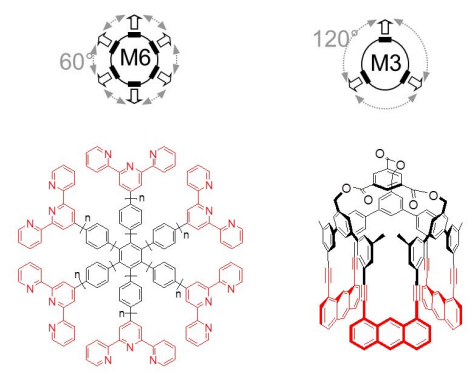

Fig. 1 Left: Two strategies that could eventually lead to 2D polymers and concrete molecular structures of monomers used for both strategies; right: the hexa-terpyridine M6 for the interfacial approach and the cyclic trisanthracene $\mathbf{M 3}$ for the single-crystal approach.

Chemistry-wise, the two approaches are based on terpyridine (tpy) metal complexation $[18,19]$ and photoinduced anthracene/acetylene [4+2]-cycloaddition [20]. We will first focus on the interfacial approach using monomer M6 $[8,21]$ and tpy complexation and then on the single-crystal work. Two aspects had to be investigated first: (i) does monomer M6 spread at the air/water interface and does it do so by lying flat on the interface and (ii) is there a read-out for successful complexation. Point (i) was addressed by measuring the surface pressure isotherm which resulted in a mean molecular area of approximately $520 \AA^{2}$, a value in good agreement with the monomers lying flat. This was confirmed by atomic force microscopy (AFM) scratching experiments in which a monolayer transferred onto mica substrate was scratched with the AFM tip in contact mode. If the scratched area was then imaged in tapping mode, a step-height of approximately $0.8 \mathrm{~nm}$ was observed, which is much closer to the proposed monolayer structure rather than a layer in which the disc-like monomer is tilted upwards relative to the surface plane. Point (ii) was addressed by using a water-soluble model tpy (structure not shown) and exposing it to various metal cations including $\mathrm{Co}^{2+}, \mathrm{Ru}^{2+}, \mathrm{Zn}^{2+}, \mathrm{Pb}^{2+}, \mathrm{Ni}^{2+}$, and $\mathrm{Fe}^{2+}$. Of all these ions, only $\mathrm{Fe}^{2+}$ gave an intense metal-to-ligand charge-transfer band (MLCT) in the UV spectrum and was therefore selected for the first orienting studies. Obviously, other metal ions may eventually be more attractive candidates (e.g., $\mathrm{Zn}^{2+}$ or $\mathrm{Cd}^{2+}$ for highly reversible networks [22] and $\mathrm{Ru}^{2+}$ for irreversible networks), but in this initial phase of the project we felt the need to have an as easy as possible handle to assess whether complexation had occurred. Using $\mathrm{Fe}^{2+}$ with its intense MCLT we could even imag- 
ine monitoring the reaction progress directly at the interface in transmission. Figure 2 shows a typical set-up and the UV spectroscopic response of a compressed monolayer (typically $2 \mathrm{nN} / \mathrm{m}$ ) upon addition of $\mathrm{Fe}\left(\mathrm{NH}_{4}\right)_{2}\left(\mathrm{SO}_{4}\right)_{2}$ to the subphase. Already within a few minutes, curve 1 showed a weak signal in the expected range, the intensity of which turned quickly constant. For comparison, Fig. 2 also shows the UV-vis spectrum of the above-mentioned model tpy when complexed with $\mathrm{Fe}^{2+}$ in a 2:1 complex and a spectrum of the monolayer after its transfer onto glass. Note that in the latter case, the intensity of the MLCT is much higher because of the significant losses of intensity in the in situ experiments caused by the optical set-up used. We consider the excellent match between the UV-vis spectra of model complex and the one at the interface (before and after transfer) as strong indication that bond formation between the monomers has taken place.
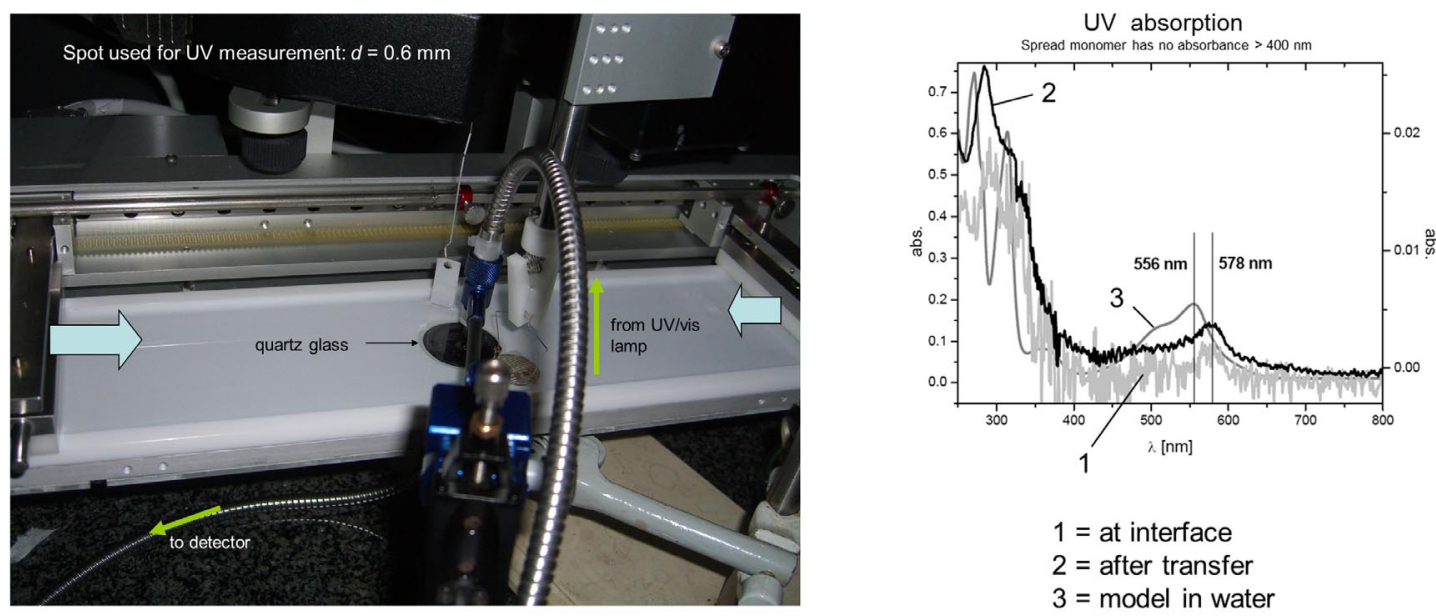

Fig. 2 The interfacial set-up and the UV-vis spectra recorded directly at the interface (line 1), after transfer of the complexed monolayer onto mica substrate (line 2) and of the water-soluble tpy model compound (line 3). The setup consists of a Langmuir-Blodgett trough with a quartz window in its bottom and fiber optics that allow the UV-vis spectra to be measured directly through the interface.

After having gathered evidence for connection between the monomers M6 at the interface, it was an obvious next task to see whether the connected monolayer is strong enough to be spanned over large holes and to also prove that the monolayer without metal salt is not strong enough for the same. This would qualitatively substantiate network formation. Figure 3 shows a transmission electron microscopy (TEM) micrograph of a monolayer after exposure to $\mathrm{Fe}^{2+}\left(0.1 \mathrm{mM}\right.$ final $\mathrm{Fe}^{2+}$ in the subphase $)$ and after horizontal Schäfer transfer onto a $\mathrm{Cu}$ grid with approximately $20 \times 20 \mu \mathrm{m}^{2}$ squared holes. While the film shows clear signs of rupturing, large portions of it are fully intact. Further, the film shows tablecloth effects, which are an important sign for mechanical integrity. Gratifyingly, the control experiment, that is when the monolayer without metal exposure was transferred, despite numerous attempts no spanned holes (not even partially) could be observed. Actually, there was nothing to be seen. The monomers obviously fell through the holes upon removal from the interface. 


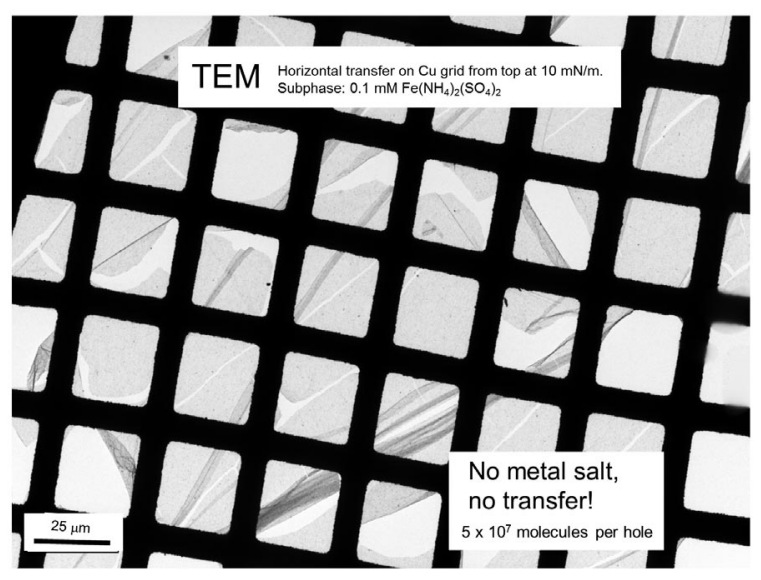

Fig. 3 TEM micrograph of a film of monomer M6 horizontally transferred from the air/water interface after exposing it to $0.1 \mathrm{mM} \mathrm{Fe}\left(\mathrm{NH}_{4}\right)_{2}\left(\mathrm{SO}_{4}\right)_{2}$ from the subphase. Reproduced with permission from ref. [21].

With the help of AFM and optical microscopy $(\mathrm{OM})$ imaging, the claim was further substantiated (not shown) [21]. Tapping-mode AFM imaging (TM AFM) showed homogeneous films with a layer thickness of 1.3-1.4 nm, which is in good agreement with the calculated monolayer thickness of $\sim 0.8$ $\mathrm{nm}$ plus counter ions $\left(\mathrm{SO}_{4}{ }^{2-}\right)$ and possibly some residual water. The AFM images also show tablecloth effects with the multiple layers always having thicknesses which are multiples of a monolayer. Information regarding the lateral extension of the films could be gained from OM of samples that had been vertically transferred onto $300 \mathrm{~nm} \mathrm{SiO}_{2}$ on $\mathrm{Si}$ wafers. OM images (not shown) show homogeneous films with dimensions in the millimeter range!

While the internal molecular structure still needs to be proven, the interfacial approach using monomer M6 and metal complexation provides for the first time access to monolayered metal-coordination polymers that are mechanically stable enough to be free-standing. Several other approaches had not led to this point.

The second approach rests upon monomer M3 and its photoinduced polymerization in the single crystal [23]. For that purpose, suitable single crystals were grown and analyzed for their internal structure. Of particular interest was whether the monomer would crystallize in layers and whether the reactive groups (anthracene/anthracene or anthracene/acetylene) would be in sufficiently tight distance so as to ideally allow for a topochemical reaction [24] to take place between them. Hexagonally shaped single crystals of $\mathbf{M 3}$ were grown from a tetrachloroethane/THF mixture. Diffraction proved the monomers to form a layered structure in which consecutive monomers are oriented upside down such that the 9,10 positions of the anthracene unit of one monomer are in tight contact ( 4.5 and $3.6 \AA$ ) with the acetylene unit of the neighboring monomer. This in principle should allow for a photochemical reaction between all the monomers which, irrespective of its actual mechanism, is confined to the sublayers within the single crystals, which consist of the reactive parts. These reactive sublayers are separated from one another by nonreactive ones that are built from the terphenylene bridges of the monomers. Irradiation of the single crystals under exclusion of oxygen not only led to a gradual disappearance of monomer fluorescence but also to a severely altered solubility behavior. While the non-irradiated monomer crystals dissolve in $N$-methylpyrrolidone (NMP) within a few minutes, the irradiated do not under such conditions.

This severely altered solubility behavior was a strong indication for a reaction in the crystal. This was further substantiated by exfoliation experiments that in the beginning only led to packages of sheets. These packages, however, had very sharp edges and also vertices reminiscent of the crystals from which they were obtained. While these packages were obviously not the ultimate goal, they played 
an important role in structure characterization. Thin organic matter directly exposed even to relatively weak electron beams is extremely sensitive and tends to "burn away". For structure analysis by electron diffraction, it was therefore desirable to have an aggregate of several sheets that is thick enough to withstand the electron beam for some time and at the same time is not too thick so as to not allow the beam to pass through anymore. This is where these packages turned out to be ideal. TEM images in unfiltered and filtered form of such a package showed rather regular hexagonal features on the length scale of the monomer. To confirm that this regular appearance was not an artifact, several such objects were subjected to electron diffraction under cryogenic conditions, which gratifyingly led a diffraction pattern to which a molecular model based on the monomers could be fitted. From this moment on we were sure to have a layered structure with internal periodicity of the constituents. All that was still needed to be proven was to show that the packages could be exfoliated down to the single sheet and to determine the connection chemistry.

Both goals could be achieved. The first exfoliation experiments had been done under relatively mild conditions (e.g., refluxing chloroform). As soon as more forcing conditions were applied (e.g., $150{ }^{\circ} \mathrm{C}$ NMP), single sheets could actually be obtained. In addition to a TEM analysis of such sheets spanned over the holes of a lacey carbon-coated $\mathrm{Cu}$ grid, AFM analysis of sheets prepared on mica proved their monolayered covalent structure. Furthermore, the match between AFM height and model is convincing and can be considered final proof for having achieved a covalently connected internally regular, monolayer sheet, or in other words, a 2D polymer. Some of the sheets on mica show straight edges and vertices as one would expect from sheets exfoliated from hexagonal crystals. At this point, the internal structure of the sheets suggested itself by the proximity of the reactive sites in the monomer crystal and the fact that any cross-linking reaction would be restricted to the sublayer containing the anthracene/acetylene moieties; a firm proof for this, however, was still missing. This is where Raman spectroscopic measurements came in. When performed on irradiated crystals they showed the expected disappearance of the two characteristic anthracene signals at $\sim 1380$ and $\sim 1560 \mathrm{~cm}^{-1}$ and the reduction of the intensity of a typical acetylene signal at $2200 \mathrm{~cm}^{-1}$ by roughly $50 \%$. Since each monomer contains three anthracenes and six acetylenes, this was the expected finding for a $[4+2]$-cycloaddition to take place.

\section{SUMMARY AND OUTLOOK}

What has been achieved? No less a figure than Hermann Staudinger in the 1920s established the term "Makromolekül" or "Polymer" as an "infinitely" long covalent chain molecule whose structure consists of sequence of (typically alike and linear) repeat units [6]. Based on this concept, which truly changed the world, we claim to have achieved the first passable trail into polymers with areal repeat units (Fig. 4). Monomers do not combine to ultrathin threads anymore but rather to ultrathin sheets with internal periodicity.

We are convinced that this finding will stimulate polymer physics but also eventually lead to novel applications. An immediate question is inasmuch linear polymers differ from areal ones? Of linear polymers it is known that they coil in solution and in melt; it is known that they entangle and that this causes all the properties that are commonly associated with plastic materials. Finally, it is known that it comes to chain-chain stacking when they crystallize. A prominent example here is chain-folded polyethylene. None of these fundamental features of linear polymers can possibly be expected for sheets. 2D polymers cannot coil but will crumple instead; they cannot entangle but may rather aggregate in crumpled form and they cannot exhibit chain-chain contact but may rather be forced into face-face contact. These differences are so pronounced that it will take a while until all the consequences are understood that these structural differences will have. An important prerequisite for this development to really set off will be the accessibility of sheets in large quantity. The single-crystal experiments described above were typically done on a few milligrams scale. It is therefore of utmost importance to devise synthetic routes that allow generating sheets at least on the g-scale. Given the simple crystallizability of monomer M3 

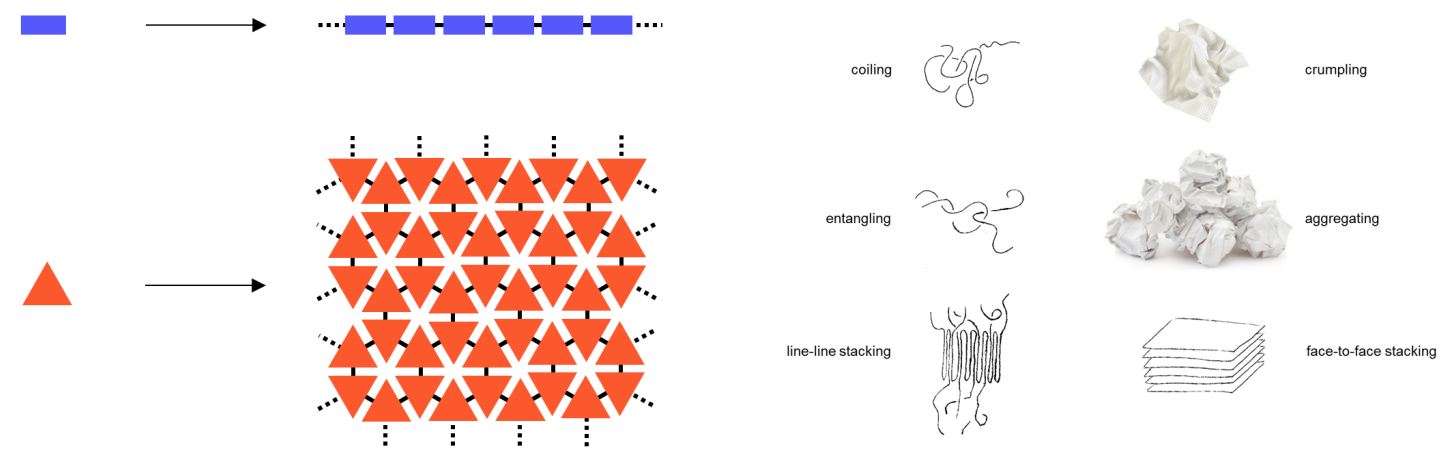

Fig. 4 Left: Linear vs. areal repeat units in linear polymers and 2D polymers. Right: Some fundamental properties in which these two kinds of polymers differ from one another.

we feel that the single-crystal approach may offer this. In terms of applications, 2D polymers open up several options, a few of which should be mentioned. Supposing their structures have little defects, their use as molecular sieves, as ultrasensitive pressure-sensors, or for surface coatings comes to mind. Other options include molecular landscaping, rational catalyst design, and strong and tough materials. The future for $2 \mathrm{D}$ polymers is on.

\section{ACKNOWLEDGMENTS}

We cordially thank all our hard-working and enthusiastic co-workers who helped us make this happen. Particularly, we wish to thank the doctoral students Thomas Bauer and Patrick Kissel who were the major players in the above projects. The $2 \mathrm{D}$ polymer project could not have been carried outout with the very engaged and competent support by external collaboration partners who include: Dr. W. B. Schweizer, Mr. M. Solar, Mr. J. Rabl, Dr. T. Tomizaki (PSI, Villigen), Dr. T. Weber, and Prof. W. Steuer for X-ray crystallography; Dr. R. Erni (EMPA, Dübendorf) and Dr. M. D. Rossell for TEM; Mr. R. Enning and Prof. A. Stemmer for AFM; Dr. A. Renn, Dr. S. Götzinger, and Prof. V. Sandoghdar (MPIPL, Erlangen) for help with UV/vis and fluorescence spectroscopies; A. Eyssler (EMPA, Dübendorf) for Raman spectroscopy; Dr. K. Feldman and Prof. P. Smith for OM; Prof. B. T. King (University of Nevada, Reno) for computation and discussion. Finally, we wish to thank Prof. G. Wegner (MPI-P, Mainz) for helpful discussions and his interest in this work. The authors thank the ETH and the Swiss National Science Foundation for financial support (TH 05 07-1, ETH-26 10-2, and SNF 200021129669).

\section{REFERENCES}

1. J. Sakamoto, J. van Heijst, O. Lukin, A. D. Schlüter. Angew. Chem., Int. Ed. 48, 1030 (2009).

2. G. Gee, E. K. Rideal. Proc. R. Soc. London, Ser. A: Math. Phys. Sci. 153, 116 (1935).

3. G. Gee. Proc. R. Soc. London, Ser. A: Math. Phys. Sci. 153, 129 (1935).

4. K. S. Novoselov, A. K. Geim, S. V. Morozov, D. Jiang, Y. Zhang, S. V. Dubonos, I. V. Grigorieva, A. A. Firsov. Science 306, 666 (2004).

5. A. K. Geim, K. S. Novoselov. Nat. Mater. 6, 183 (2007).

6. H. Staudinger. Ber. 53B, 1073 (1920).

7. P. Kissel, A. D. Schlüter, J. Sakamoto. Chem.-Eur. J. 15, 8955 (2009).

8. T. Bauer, A. D. Schlüter, J. Sakamoto. Synlett 877 (2010).

9. D. F. Perepichka, F. Rosei. Science 323, 216 (2009). 
10. M. J. Schultz, X. Zhang, S. Unarunotai, D.-Y. Khang, Q. Cao, C. Wang, C. Lei, S. MacLaren, J. A. N. T. Soares, I. Petrov, J. S. Moore, J. A. Rogers. Proc. Natl. Acad. Sci. USA 105, 7353 (2008).

11. M. Bieri, M. Treier, J. Cai, K. Ait-Mansour, P. Ruffieux, O. Gröning, P. Gröning, M. Kastler, R. Rieger, X. Feng, K. Müllen, R. Fasel. Chem. Commun. 6919 (2009).

12. K. T. Nam, S. A. Shelby, P. H. Choi, A. B. Marciel, R. Chen, L. Tan, T. K. Chu, R. A. Mesch, B.-C. Lee, M. D. Connolly, C. Kisielowski, R. N. Zuckermann. Nat. Mater. 9, 454 (2010).

13. M. Abel, S. Clair, O. Ourdjini, M. Mossoyan, L. Porte. J. Am. Chem. Soc. 133, 1203 (2011).

14. I. Berlanga, M. L. Ruiz-González, J. M. González-Calbet, J. L. G. Fierro, R. Mas-Ballesté, F. Zamora. Small 7, 1207 (2011).

15. Q. An, Q. Chen, W. Zhu, Y. Li, C.-A. Tao, H. Yang, Z. Li, L. Wan, H. Tian, G. Li. Chem. Commun. 46, 725 (2010).

16. J. M. Cai, P. Ruffieux, R. Jaafar, M. Bieri, T. Braun, S. Blankenburg, M. Muoth, A. P. Seitsonen, M. Saleh, X. L. Feng, K. Müllen, R. Fasel. Nature 466, 470 (2010).

17. M. Bieri, M. T. Nguyen, O. Groning, J. M. Cai, M. Treier, K. Ait-Mansour, P. Ruffieux, C. A. Pignedoli, D. Passerone, M. Kastler, K. Müllen, R. Fasel. J. Am. Chem. Soc. 132, 16669 (2010).

18. U. S. Schubert, H. Hofmeier, G. R. Newkome. Modern Terpyridine Chemistry, Wiley-VCH, Weinheim (2006).

19. P. R. Andres, U. S. Schubert. Adv. Mater. 16, 1043 (2004).

20. H. D. Becker, K. Andersson, K. Sandros. J. Org. Chem. 50, 3913 (1985).

21. T. Bauer, Z. Zheng, A. Renn, R. Enning, A. Stemmer, J. Sakamoto, A. D. Schlüter. Angew. Chem., Int. Ed. 50, 7879 (2011).

22. J.-L. Wang, X. Li, X. Lu, I.-F. Hsieh, Y. Cao, C. N. Moorefield, C. Wesdemiotis, S. Z. D. Cheng, G. R. Newkome. J. Am. Chem. Soc. 133, 11450 (2011).

23. P. Kissel, R. Erni, W. B. Schweizer, M. D. Rossell, B. T. King, T. Bauer, S. Götzinger, A. D. Schlüter, J. Sakamoto. Nat. Chem. 3 (2012). <http://dx.doi.org/10.1038/NCHEM.1265>

24. G. Wegner. Z. Naturforsch., B: Chem. Sci. 24, 824 (1969). 